\title{
Healthy Pacific grandparents: a participatory action research project exploring ageing well amongst Pacific people in New Zealand
}

El-Shadan Tautolo, ${ }^{\mathrm{a} * 1}$ Wendy Wrapson, ${ }^{\mathrm{a}}$ Janis Paterson, ${ }^{\mathrm{a}}$ Valerie Wright-St Clair, ${ }^{\mathrm{b}}$ Stephen Neville ${ }^{\mathrm{b}}$ Ofa Dewes, ${ }^{\mathrm{c}}$ and Leon Iusitini ${ }^{\mathrm{a}}$

${ }^{a}$ Centre for Pacific Health \& Development Research, Auckland University of Technology, Auckland, New Zealand; ' Centre for Active Ageing, Auckland University of Technology, Auckland, New Zealand; 'School of Nursing and Maurice Wilkins Centre for Molecular Discovery, Faculty of Medical \& Health Sciences, University of Auckland, Auckland New Zealand

The New Zealand older adult population (aged 65+ years) is growing at a faster rate than the younger population, with many of those in the later years living much longer. The proportion of older Pacific people is forecast to reach $4.1 \%$ of the country's total population within the next two decades, highlighting the importance of research focussed on ageing Pacific populations. Objective: This paper sets out the research protocol and methods for The Pacific Islands Families: Healthy Pacific Grandparents' Study which aims to investigate older Pacific peoples' viewpoints on ageing to identify specific cultural values, perspectives and understandings as the Pacific population in New Zealand ages. Discussions will focus upon key themes including: (1) Engagement with family, the community, and health service provision; (2) Support within their day-to-day lives; (3) Intergenerational living patterns and relationships; and (4) physical and psychosocial barriers to meaningful participation. Design: The study will recruit and utilise participants from a grandparent cohort that is nested within the families of the longitudinal Pacific Islands Families Study. This study uses a Participatory Action Research approach to position the participants in a leadership role where they are co-researchers involved in both the research and the implementation of recommendations. Implications: Findings will help determine whether current social and health system environments meet the needs of older Pacific people, and devise and implement solutions to deal with identified deficits. Utilising a transformative research process will bring older Pacific people together to define for themselves their needs and their experiences, identify any areas of shortcoming, and support the implementation of solutions through strategic and informed actions.

Keywords: Pacific; grandparents; social; connected; health; ageing; co-design

\section{Introduction}

The New Zealand (NZ) older adult population (aged 65+ years) is growing at a faster rate than the younger population, with many of those in the later years living with some form of disability (Jantrana \& Blakey, 2008). While Pacific people only made up $2.4 \%$ of the total NZ population aged 65 and over in the 2013 NZ Census of Population and Dwellings, this proportion is forecast by Statistics New Zealand to increase by $70 \%$ to reach $4.1 \%$ within the next two decades, highlighting the importance of research focussed on health and wellbeing in ageing Pacific populations. This is especially so given several recent reports (Ministry of Health, 2012;

1 * Corresponding author El-Shadan Tautolo, Email:dan.tautolo@aut.ac.nz 
Ministry of Social Development, 2010; Southwick, Kenealy, \& Ryan, 2012) showing little improvement in the socioeconomic circumstances of Pacific people, and little change in their overall health status, while further research findings from Richardson and colleagues (2013) indicate mortality rates have decreased in all ethnic groups in NZ except Pacific people where they have remained the same.

\section{Existing research on older Pacific people}

Despite little prior research in NZ on ageing and older Pacific people, two previous studies have attempted to incorporate Pacific perspectives within the context of ageing research. One study from Wiles and colleagues (2011) involved a diverse group of NZers, including older Samoans and Cook Islanders. The research identified six principles for resilient ageing, including the necessity for policies, practices, and strategies to be developed in partnership with older people and to leverage and bolster their skills and expertise in advocacy. Similarly, another study from Tamasese et al. (2014) used focus groups to explore older Pacific peoples' perspectives on cultural concepts of ageing, differences between mainstream and Pacific perspectives, living standards, and social inclusion.

However, although these studies included older Pacific people as participants, they did not specifically explore the topic of ageing or the health and wellbeing of older Pacific people. Pacific people, who comprise $7.4 \%$ of NZ's population (295,941 people), and are the fourth largest ethnic group in NZ (Statistics New Zealand), are entitled to experience the same level of health and wellbeing as non-Pacific people. However, current health disparities show this is not the case. 90\% of Pacific people in New Zealand live in low decile areas with significant social and economic disadvantage. Socioeconomic disadvantage is closely correlated with poor health and access to health services (Tukuitonga, 2013).

Unsurprisingly, Pacific people are more likely to have unmet primary health care needs when compared to non-Pacific people. Barriers to accessing health care include, not visiting a General Practitioner due to cost or due to lack of transport, and inability to collect a prescription due to cost, with rates almost triple for Pacific adults compared with non-Pacific adults (Ministry of Health, 2015b). Pacific adults also have low rates of visiting a primary health care nurse (Ministry of Health, 2015b), despite these nurses playing an integral role in health promotion and wellness programmes. Furthermore, although Pacific adults have a low rate of diagnosed mood and/or anxiety disorders, they are more likely to have experienced high levels of psychological distress in the past four weeks which could indicate anxiety or depressive disorders (Ministry of Health, 2015b). Such data suggest differences in health service provision and use of health services by Pacific people.

\section{Social engagement and health outcomes}

There is substantial international evidence that active social engagement of older people is associated with physical, cognitive and psychological health benefits (Adams, Leibbrandt, \& Moon, 2011; Bath \& Deeg, 2005; Rozanova, Keating, \& Eales, 2012; Walker et al., 2013; Warburton \& McLaughlin, 2005), as well as being associated with a lower risk of mortality (Maier \& Klumb, 2005), providing a greater sense of purpose and self-efficacy (de Leon, Glass, \& Berkman, 2003), and increasing a person's chances of receiving needed support (Cornwell, Laumann, \& Schumm, 2008). Social engagement is normally defined as involvement in activities which have a social element (Bath \& Deeg, 2005) but, in the current context, we characterise 'social engagement' more broadly as also encompassing 'social connectedness' (the relationships people have with others) and interaction with health services.

Taking this broader stance, NZ research reveals that: (1) Pacific people have poorer health and greater unmet health needs than the non-Pacific population (Ministry of Health, 2015); (2) opportunities for interactions with health service providers may be limited for Pacific people 
due to financial and language barriers, and mono-cultural assumptions and practices of health care professionals (Ministry of Health, 2012; Tamasese et al., 2014); and (3) older people, including those identifying as Pacific, acknowledge interacting with others is a key aspect of maintaining wellbeing and resilience in older age (Tamasese et al., 2014).

Pacific health models and cultural beliefs, values and practices suggest 'wellness' is holistic, and comprises physical, mental, social, and spiritual dimensions; with strong family relationships (Tamasese et al., 2014); and health is a family concern rather than an individual matter (Laing \& Mitaera, 1994). There may be a reluctance to hand over family members for care because they become separated from their family in an unfamiliar environment and because clinicians deal primarily with the individual concerned, whereas illnesses for Pacific people are family matters (Bathgate \& Pulotu-Endemann, 1997). Traditional Pacific healers and complementary therapies may therefore be used in addition to, or in place of, conventional treatment (Pack, Minster, Churchward, \& Tanuvasa, 2013), particularly by older people. Moreover, it has been suggested the traditional respect for authority figures in Pacific communities (Statistics New Zealand and Ministry of Pacific Island Affairs, 2010) can make it difficult for Pacific people to demand more culturally appropriate and effective services. Alongside this, the influence of religious beliefs on the perspectives of older Pacific people regarding services and solutions, should also be explored.

Social cohesion may protect against some of the adverse effects of ageing-related health conditions (Statistics New Zealand and Ministry of Pacific Island Affairs, 2011); significant associations have been demonstrated between social disconnectedness and loneliness, poorer physical health, and mental health, particularly for minority groups. Pacific people have traditionally demonstrated high levels of social connectedness, frequently having extensive family networks, and taking an active role in Pacific community groups, church life and volunteering. Church has been considered by some older Pacific people to be a village away from the islands (Macpherson, 1996) where Pacific families embrace social connections, maintain social support and where cultural and religious needs are met (Anae, 2001; Tiatia, 1998).

In addition to religion playing an important role in social connectedness (Manuela \& Sibley, 2013), an individual's identity and well-being are also traditionally dependent on family heritage, roles, connections, and responsibilities within their community. Historically, older people have held a vital social role by preserving and passing on cultural traditions and values to younger generations (Ihara \& Vakalahi, 2012), but the composition of families is changing. It is unclear what impact delayed marriage, dual-career families, intergenerational living arrangements, and caring for grandchildren (Worrall, 2009) is having on older Pacific people's roles in kinship networks, and on wider social involvement.

\section{Participatory action approach}

Participatory Action Research (PAR) is a methodology supported by a growing body of international literature that is effective in providing innovative solutions to a range of issues in varying community and ethnic groups (Blair, 2009; de Santana \& Neto, 2015; Hogan et al., 2014; MacKenzie, Tan, Hoverman , \& Baldwin, 2012; Panelli, Gallagher, \& Kearns, 2006; Patten, Mitton, \& Donaldson, 2006). PAR studies hold promise for helping to address and understand some of the challenges faced by older minority people, as well as other marginalized groups (Blair, 2009; Ericson-Lidman \& Strandberg, 2015; Moreno-John et al., 2004).

However, although PAR is increasingly viewed as an important complement to traditional investigator-driven research, relatively little PAR has been undertaken where older adults have played a prominent role as research partners (Blair, 2009). Nevertheless, PAR approaches have been used in international studies with older people to enhance stroke services through user 
involvement (Jones, Auton, Burton, \& Watkins, 2008), to identify and eliminate fall risk hazards (Gallagher \& Scott, 1997), to identify effective interventions for people with early dementia (Nomura et al., 2009), and to identify and address issues of concern of retirement village residents (Ritchie, Bernard, Trede, Hill, \& Squires, 2003).

The proposed study seeks to position Pacific people in a leadership role where they are coresearchers involved in all aspects of both the research and the implementation of recommendations. The PAR process also holds promise for helping to understand the role culture plays in contributing to the wellbeing of older Pacific people and in addressing some of the complex health and social problems faced by this population.

\section{Proposed research}

The proposed research aims to investigate Pacific viewpoints on ageing to identify specific cultural values, perspectives and understandings that will become increasingly important for relevant community, social and health services as the Pacific population in NZ ages. The broader research question is: How does meaningful engagement and participation in later life contribute to healthy ageing among older Pacific people?

The first objective of the study will be to define what older Pacific people interpret 'meaningful engagement and participation' to mean in the context of their own lives, and what is their perspective on 'healthy ageing' in relation to their own chronological age and health status? Following this, we propose discussion around four potential themes: (1) the different ways in which older Pacific people engage with family, the community, and health service provision; (2) the extent to which older Pacific people feel supported in their day-to-day lives and the value they place on the different interactions that occur; (3) the extent and significance of intergenerational living patterns and relationships; and (4) physical and psychosocial barriers to meaningful participation. These themes can be added to, or changed, as deemed appropriate by our PAR co-researchers.

\section{Methods \\ Study design}

The Pacific Islands Families: Healthy Pacific Grandparents (PIF:HPG) Study is a PAR project, with a new grandparent cohort, that is nested within the families of the longitudinal PIF Study (Paterson et al., 2008). The PIF Study is an ongoing longitudinal study of Pacific children born at Middlemore Hospital, South Auckland, NZ, between March and December 2000. In addition to information collected from the children, data concerning family functioning and the health and development of the child have been collected from mothers at eight, and fathers at six, different time-points thus far. Detailed information about the PIF cohort and procedures is described elsewhere (Paterson et al., 2008).

The PIF:HPG study will engage with the grandparent generation to identify, and initiate solutions to address, what matters to older Pacific people in relation to social participation and healthy ageing. For a community research initiative to be successful over the long term, the issues to be addressed must be identified by the community and be viewed as a priority that compels action (Hogan et al., 2014; Montesanti, Abelson, Lavis, \& Dunn, 2015) One of the most important features of PAR is that it enables communities and researchers to work together to develop an understanding of issues and to take actions that will support culturally appropriate and effective strategies (Harrison \& Graham, 2012). PAR work has been successfully used to partner with marginalised and indigenous groups and offers a way to make the research meaningful for a community (Kemmis \& McTaggart, 2005), being based on an action cycle that assists in improving processes for addressing issues from the communities' perspective (McIntyre, 2008). The PAR design will guide grandparent research partners through an action- 
reflection cyclical process of (1) Issue identification and clarification, (2) Solution building, and (3) Implementation, Evaluation, and Nurturing the Change.

\section{Participants}

Sixty-six families will be recruited from the PIF Study cohort based on ethnicity (Samoan, Tongan, and Cook Islands Māori), gender, health status (Robinson, 2014), and place of birth. It is important to note that inclusion of the smaller Pacific ethnic groups in the PIF:HPG is not feasible due to the qualitative design, the size of the sample, and the construction of the PAR groups. Purposeful qualitative sampling using a maximum variation strategy (Maxwell, 2005) will be used to guide the selection and recruitment of participatory action research groups. This is based on our interest in gaining diverse perspectives and experiences about the needs and expectations of older Pacific people in relation to the social and health contexts in which they live in NZ. The underlying principle is to select information-rich cases, from which one can learn about the issues of central importance to the research questions (Coyne, 1997). The existing PIF Study provides a unique sampling frame for the PIF:HPG Study.

Recruitment will be carried out through the consent of families in the longitudinal PIF Study living in Auckland. Selected families will be invited to nominate a grandparent of the cohort child, who also lives in Auckland, as a potential participant in the PIF:HPG Study. The Pacific ethnic group(s) of the family will be determined through the existing PIF database but the gender, health status, and place of birth of the grandparent will be explored in this first contact with the family and will confirm eligibility of the grandparent for the PIF: HPG Study. When the identified grandparent has agreed to talk to a researcher, a Pacific researcher will visit the grandparent to describe the study, discuss their collaboration as a research partner, and gain informed consent. Participant information sheets and consent forms will be available in the relevant Pacific languages, in addition to versions in English. At this time conversations with the grandparent participants will focus on explaining the cyclic nature of the PAR process of planning, acting, observing, and reflecting to make sure that the participants feel comfortable about their role as research partners (McIntyre, Chatzopoulos, Politi, \& Roz, 2007).

In preparation for the PIF:HPG Study, consultations with grandparents has taken place to 'set the scene' for the study and to begin the identification of issues that face Pacific grandparents. Similarly, there has been discussion of the study with relevant stakeholders to garner support for the implementation phase of the study. While there is no specific age limit for participants wishing to take part in the study, it must be acknowledged that the NZ pacific population is very young with very high fertility rates and high levels of teenage pregnancy. As a consequence, there is a strong possibility that 'grandparents' identified and recruited for the study may be much younger than grandparents from other ethnic groups. However, we do not view this as a weakness, and instead consider it an opportunity to explore other relevant issues for ageing well Pacific communities, such as the sole custody and care of grandchildren, and the impact of traditional gift-giving on social engagement or participation.

\section{Three phases of PAR Process}

The PIF:HPG Study will undertake the first two phases of the PAR process, (1) Issue Identification and Clarification, and (2) Solution Building in 2017, and the third phase (3) Implementation, Evaluation, and Nurturing the Change in 2018 (see Figure 1).

\section{Phase 1. Issue Identification and Clarification}

Issue identification by participants, rather than by researchers, (see figure 1) forms the foundation of the study and is reliant upon the grandparents sharing their knowledge and expertise in developing valid and authentic aspirational, yet realistic, outcomes. During this first phase partnerships are formed between researchers and participants that will create a 
Talanoa and Talanga are two Pacific-centred methods or approaches for collecting and discussing information with Pacific people and communities. Although more commonly implemented with Tongan participants, certain features are relevant for other Pacific ethnic groups, supporting their broader utility. According to Vaioleti (2006), Talanoa can be referred to as a conversation, a talk, an exchange of ideas or thinking, whether formal or informal. Tala means to inform, tell, relate and command, as well as to ask or apply, while Noa means of any kind, ordinary, nothing in particular, purely imaginary or void. The Talanoa approach involves talking things over rather than taking a rigid stand, and incorporates oratory and verbal negotiations which, according to the NZ Ministry of Education (2001), have deep traditional roots in Pacific cultures. Therefore, in Talanoa researchers are flexible and open to whatever participants choose to share with them. Similarly, Ofanoa and colleagues (2015) characterise Talanga as interactive talking with a purpose, which embraces cultural values to engage with participants in a meaningful and appropriate way.

reliable, comprehensive, and shared understanding of the issues that affect them as they age, and clarify the research path. Three ethnic-specific (Samoan, Tongan, and Cook Islands Māori) participatory action research groups (PARGs) will be formed comprising grandparent research partners. Within each ethnic PARG there will be four subgroups (5-6 grandparents in each group). The structure of the subgroups will be decided by each ethnic-specific PARG, and may include a mix of male-only, female-only, and/or mixed gender groups.

The overall project will require strong cultural competency, guidance, and involvement, and the recruitment and establishment of a Pacific reference group, is an essential facet for achieving this aspiration. In addition, Pacific researchers, trained in participatory action research group methodologies and conversant in the relevant Pacific language, will be ethnically-matched to facilitate the twelve PARG subgroups, and identify issues and gather perspectives on the research objectives. All focus groups will be held at the AUT South campus and will be approximately two hours in duration. Transport and refreshments will be available on every occasion.

Talanoa (Vaioleti, 2006) and Talanga (Ofanoa et al., 2015) research methods will be used to allow for all PARG group members' voices to be heard. The interaction, which will be audiorecorded, will be guided by how participants describe meaningful engagement and participation in their lives. The broader research question will assist the PAR groups to consider their engagement with family, community, and health service provision, their feelings of support, and things they value in their daily lives. An exploration of the physical and psychosocial barriers to meaningful participation and the actions that could be taken to make a difference to older Pacific peoples will form the basis of the solution building phase. Throughout the action research groups, two-way interaction between researchers and grandparents will be enabled and the grandparents' roles as partners in the solution building and implementation phases will be clarified and developed. For example, a subgroup may wish to engage intergenerational family members in a discussion, and to feed-back their perspectives to the subgroup for consideration. Amendments to ethics approval would be gained for these possible changes in process, as required. After each of the twelve PARG subgroup discussions, the data will be transcribed verbatim. Preliminary analysis will be undertaken by researchers who will then take it back to the subgroups for discussion. Collaborative descriptive and interpretive accounts of the focus group data will be developed by the research partners (researchers and grandparents) within each subgroup in a workshop setting. Following on from this workshop, the data will be coded inductively using thematic analysis, and incorporating a holistic Pacific framework and approach to interpretation (Bryman, 2015). 


\section{Solution building}

Based on themes and recurring ideas and concepts identified in the data (Gerber, 1999), the research partners in each subgroup will focus on what steps should be taken to get the best outcomes. This is a proactive exchange to identify the way forward to improve social participation and healthy ageing for Pacific peoples. When the generation of key themes and the process of building solutions in each of these twelve ethnic-specific subgroups is completed (see Figure 1), then these subgroups will be collapsed into the three ethnic specific participatory action research groups ( $\mathrm{n}=22$ in each). The key themes/proposed solutions from the subgroups will then be presented back to the relevant ethnic specific group to obtain consensus and ratification. This will be a critical process of weaving together the different expressions of knowledge and ideas from within the various ethnic groupings. At this time two grandparent representatives will be nominated to represent their ethnic group in the implementation process with stakeholders and families (six representatives in total). A summary report of the confirmed themes and solutions will be prepared by the research team in preparation for the next PAR phase of implementation, evaluation and sustainable change.

\section{Implementation, evaluation and nurturing the change}

In this final phase, the focus is on actual implementation of the proposed solutions and an evaluation of uptake and outcomes. Planning for implementation underpins the entire process. A process evaluation is an important part of this phase and can range from a simple audit to a formal evaluation of approaches to healthy ageing of Pacific people. The input of providers and decision makers is important to evaluate what exists and what additional implementation strategies could be introduced to support Pacific grandparents. The six grandparent representatives will partner with the research team to present key themes and proposed solutions to the stakeholders and work with stakeholders and families, as guided by the ethnicspecific groups, to design the action plans. These action plans will be taken back to the full Pacific PARG ( $\mathrm{n}=66$ ) before going through to implementation. The implementation of action plans, evaluation of the actions, and development of further phases of action plans will involve grandparent research partners, individual families, and stakeholder organisations such as Vaka Tautua - a National Pacific support service for disabled and older aged people, Pacific Homecare Trust, and Aged Concern NZ. The PIF:HPG Study will be completed in 2019 when the action plans are implemented and recommended changes are judged by the research partners as effective and potentially sustainable. A twelve-month post evaluation will be carried out in 2020 through follow up interviews with the stakeholders and with a small sample $(n=10)$ of the grandparent participants. 
Figure 1: The three phases of the Participatory Action Research (PAR) process used in the PIF:HPG study
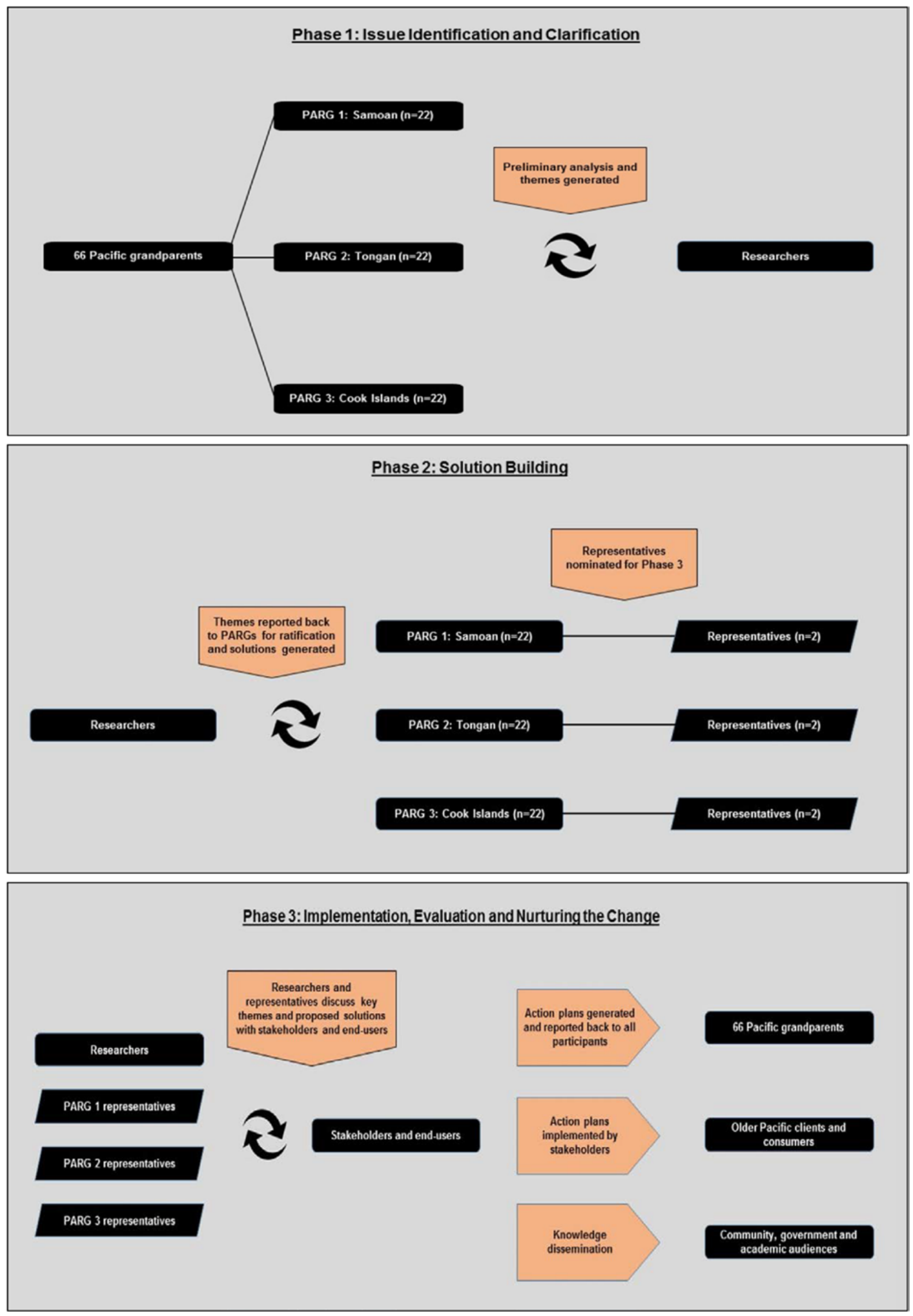


\section{Advantages of PAR research process}

A key strength is the integration of the researchers' theoretical and methodological expertise with the non-academic participants' real world knowledge and experience into a mutually reinforcing partnership. The research team believe that ageing well matters and that the best way to understand the issues important to Pacific communities is to bridge the gap between researchers and participants (Cargo \& Mercer, 2008). However it can be a demanding process that needs careful planning by the research team. PAR research within older ethnic populations can be challenging, especially with recruitment, sustained participation and language barriers (Blair, 2009; Carter, Elward, Malmgren, Martin, \& Larson, 1991; Moreno-John et al., 2004; Norris et al., 2007; Ogden, Hernandez, \& Hidalgo, 2010). Nurturance of the grandparent research partners throughout the PAR process will be required as some may struggle with their commitment to the research over time (Israel, Schulz, Parker, \& Becker, 1998). Time will be allocated to facilitate equitable participation in the process and for the timetable to be appropriate to, and supportive of, the participants. The arrangements for transport and the setup of the participatory action research groups will be focussed on the comfort and safety of the participants.

Similarly, the relationship between researchers and participants will be crucial to the success of this project. The research team must be sensitive to the participants' needs and be responsive to the different forms of leadership required at different times in the PIF: HPG Study. For example, researchers are likely to lead the data analysis whereas grandparents are likely to lead solution building and the implementation strategies. Literature suggests that in some situations, non-academic partners have limited time or interest in contributing to some technical and labour intensive components of the research process, but they want to be involved in the identification of issues, and participate in the solution building and interpretation of results (Landry, Amara, Pablos-Mendes, Shademani, \& Gold, 2006). To promote mutual respect and equitable participation, the PIF: HPG Study research team will ensure that participants are given the opportunity to participate in all phases but that they do not need to participate in every phase if they do not wish to do so (Cargo \& Mercer, 2008).

\section{Discussion}

\section{Potential outcomes}

The purpose of the proposed research is to determine whether the current social and health system environments meet the needs of older Pacific people and to devise and implement solutions to deal with identified deficits. To do this effectively, we propose a transformative research process which will bring older Pacific people together to define for themselves their needs and their experiences, identify any areas of deficit, then find solutions in conjunction with stakeholders, and to implement those solutions through strategic and informed actions. This model builds the capacity of people on the front-line of a problem to take leadership in creating the change they want.

The issues to be addressed in the proposed study, and the likely transformative outcomes, cannot yet be described because the underlying philosophy of PAR research is that it is participant-driven in terms of identifying issues that need addressing. Consequently, the specific problems to be confronted and the types of action to be taken will originate from the research process itself. However, because older Pacific people are not a homogenous group, our participant recruitment process recognises that people's needs vary at different stages of the ageing process and that it is necessary to understand the experiences of those people whose needs are relatively simple as well as those who may have unique and more complex health and social needs. In-depth information about the range of actual situations experienced by older 
Pacific people is essential for future health planning and to assist in reducing the inequities in health and wellbeing outcomes that currently exist for older Pacific people.

Previous PAR has found that interventions can continue to be ongoing after the formal work of the research has ended and that knowledge created in the process of solving problems becomes the basis for further actions (Khanlou \& Peter, 2005). Thus, transformative outcomes do not only result from the initial research project. As well as the specific actions undertaken as part of a research project, additional recommendations may be made, identifying additional issues and potential solutions that have arisen out of the research, but which were not possible to implement within the timeframe and resources available.

In addition to the outcomes directly related to the research question, PAR is an empowering process that enables participants to gain skills and competencies, thus contributing to capacity building within the community. The relationship between researchers and participants will be reciprocal, with the research team providing the skills and participants contributing their knowledge. The proposed study will make innovative use of Pacific research methodologies to allow older Pacific people's experiences to be articulated and developed as a resource to facilitate change. Talanoa and Talanga research approaches will be used, and the cyclical natural of the research will foster significant engagement and consultation by the research team with the Pacific community, which will be ongoing for the duration of the project.

Through the PIF Study, the research team is already engaged with a comprehensive group of national and local end-users and policymakers, who are in a position to utilise the information provided from the research. Interactions and consultations have commenced with organisations such as Vaka Tautua, Treasuring Older Adults \& Pacific Aiga Carers, Pacific Heartbeat, Pasifika Medical Association, NZ Association of Gerontology, Age Concern Counties Manukau, the Positive Ageing Network, and the Office for Senior Citizens, and universal support gained for the proposed PIF:HPG Study. We also connected with District Health Boards (Auckland District Health Board and Waitemata District Health Board, 2014), Primary Health Organisations, and additional Pacific service providers to identify current work being undertaken and explore areas of need as part of developing the study proposal. Throughout the project, we will build on these stakeholder relationships to foster momentum for engaging with the participatory action research process and for uptake and utilisation of research outcomes.

The PIF:HPG Study project team will be geographically situated at Auckland University of Technology's South campus in Manukau, embedding the research in the community and allowing the continuous transfer, translation and uptake of research findings in a meaningful and inclusive manner. The PIF Study team have vast experience in disseminating research findings. Approximately 150 academic publications have been generated from this longitudinal study, and the team has a proven track record in delivering practical oral and written information to end-users, resulting in support for innovative changes in service delivery and programme development. Dissemination of the research protocol through this paper is integral to the project's commitment to open communication and transparency.

\section{References}

Adams, K. B., Leibbrandt, S., \& Moon, H. (2011). A critical review of the literature on social and leisure activity and wellbeing in later life. Ageing \& Society, 31(04), 683-712. doi:doi:10.1017/S0144686X10001091

Anae, M. (Ed.). (2001). The new vikings of the sunrise: New-Zealand born in the information age. Palmerston North: Dunmore Press.

Auckland District Health Board and Waitemata District Health Board. (2014). Our health in our hands: Pacific plan for a long, healthy life 
http://www.waitematadhb.govt.nz/Portals/0/Documents/Planning/HealthActionPlans/Our HealthInOurHandsPacificHealthPlan2014_eVersion.pdf

Bath, P. A., \& Deeg, D. (2005). Social engagement and health outcomes among older people: introduction to a special section [journal article]. European Journal of Ageing, 2(1), 24-30. doi:10.1007/s10433-005-0019-4

Bathgate, M., \& Pulotu-Endemann, F. (1997). Pacific people in New Zealand. Mental health in New Zealand from a public health perspective. Wellington: Ministry of Health, 104-135.

Blair, T., Minkler, M. (2009). Participatory action research with older adults: key principles in practice. The Gerontologist, 49, 651-662. doi:10.1093/geront/gnp049

Bryman, A. (2015). Social research methods Oxford: Oxford University Press.

Cargo, M., \& Mercer, S. L. (2008). The value and challenges of participatory research: strengthening its practice. Annual Review of Public Health, 29(1), 325-350. doi:doi:10.1146/annurev.publhealth.29.091307.083824

Carter, W. B., Elward, K., Malmgren, J., Martin, M. L., \& Larson, E. (1991). Participation of older adults in health programs and research: a critical review of the literature. The Gerontologist, 31(5), 584-592.

Cornwell, B., Laumann, E. O., \& Schumm, L. P. (2008). The Social Connectedness of Older Adults: A National Profile. American Sociological Review, 73(2), 185-203. doi:10.1177/000312240807300201

Coyne, I. T. (1997). Sampling in qualitative research. Purposeful and theoretical sampling; merging or clear boundaries? Journal of Advanced Nursing, 26(3), 623-630. doi:10.1046/j.1365-2648.1997.t01-25-00999.x

de Leon, C. F. M., Glass, T. A., \& Berkman, L. F. (2003). Social engagement and disability in a community population of older adults: the New Haven EPESE. American Journal of Epidemiology, 157(7), 633-642.

de Santana, C., \& Neto, F. (2015). Developing a mental health programme for refugess based on participatory action research: an experince from Sao Paulo, Brazil. International Journal of Action Research, 11, 265-288.

Ericson-Lidman, E., \& Strandberg, G. (2015). Learning to deal constructively with troubled conscience related to care providers' perceptions of deficient teamwork in residential care of older people - a participatory action research study. Scandinavian Journal of Caring Sciences, 29, 215-224.

Gallagher, E. M., \& Scott, V. J. (1997). The STEPS Project: participatory action research to reduce falls in public places among seniors and persons with disabilities. Canadian journal of public health = Revue canadienne de sante publique, 88(2), 129-133.

Gerber, R. (1999). The role of theory in social and health research. In V. Minichiello, G. Sullivan, K. Greenwood, \& R. Axford (Eds.), Handbook for research methods in health sciences. pp. 16-33. Sydney: Pearson Education.

Harrison, M. B., \& Graham, I. D. (2012). Roadmap for a Participatory Research-Practice Partnership to Implement Evidence. Worldviews on Evidence-Based Nursing, 9(4), 210220. doi:10.1111/j.1741-6787.2012.00256.x

Hogan, L., Bengoechea, E. G., Salsberg, J., Jacobs, J., King, M., \& Macaulay, A. C. (2014). Using a Participatory Approach to the Development of a School-Based Physical Activity Policy in an Indigenous Community. Journal of School Health, 84(12), 786-792.

Ihara, E. S., \& Vakalahi, H. F. O. (2012). Collective Worldviews and Health of Pacific American Elders. Educational Gerontology, 38(6), 400-411. doi:10.1080/03601277.2011.559852 
Israel, B. A., Schulz, A. J., Parker, E. A., \& Becker, A. B. (1998). Review of community-based research: assessing partnership approaches to improve public health. Annual Review of Public Health, 19, 173-202. doi:10.1146/annurev.publhealth.19.1.173

Jantrana, S., \& Blakey, T. (2008). Ethnic inequalities in mortality among the elderly in New Zealand. Australian and New Zealand Journal of Public Health, 32, 437-443. doi:10.1111/j.1753-6405.2008.00276.x

Jones, S. P., Auton, M. F., Burton, C. R., \& Watkins, C. L. (2008). Engaging service users in the development of stroke services: an action research study. Journal of Clinical Nursing, 17(10), 1270-1279. doi:10.1111/j.1365-2702.2007.02259.x

Kemmis, S., \& McTaggart, R. (2005). Participatory Action Research: Communicative Action and the Public Sphere In N. K. Denzin \& Y. S. Lincoln (Eds.), The Sage Handbook of Qualitative Research (3rd ed.). pp. 559-603. Thousand Oaks, CA: Sage Publications.

Khanlou, N., \& Peter, E. (2005). Participatory action research: considerations for ethical review. Social Science \& Medicine, 60(10), 2333-2340. doi:http://dx.doi.org/10.1016/j.socscimed.2004.10.004

Laing, P., \& Mitaera, J. ( 1994). Samoan and Cook Islanders Perspectives on Health. In Social Dimensions of Health and Disease. New Zealand Perspectives (pp. 204-218). Palmerston North: Dunmore Press.

Landry, R., Amara, N., Pablos-Mendes, A., Shademani, R., \& Gold, I. (2006). The knowledgevalue chain: a conceptual framework for knowledge translation in health. Bulletin of the World Health Organization, 84, 597-602.

MacKenzie, J., Tan, P., Hoverman , S., \& Baldwin, C. (2012). The value and limitations of participatory action research methodology. Journal of Hydrology, 474, 11-21. doi:10.1016/j.jhydrol.2012.09.008

Macpherson, C. (Ed.). (1996). Pacific Islands identity and community. Palmerston North: Dunmore Press.

Maier, H., \& Klumb, P. L. (2005). Social participation and survival at older ages: is the effect driven by activity content or context? . European Journal of Ageing, 2(1), 31-39. doi:10.1007/s10433-005-0018-5

Manuela, S., \& Sibley, C. G. (2013). The Pacific Identity and Wellbeing Scale (PIWBS): A Culturally-Appropriate Self-Report Measure for Pacific Peoples in New Zealand. Social Indicators Research, 112(1), 83-103. doi:10.1007/s11205-012-0041-9

Maxwell, J. (2005). Qualitative Research Design: An interactive approach. Thousand Oaks, CA: Sage.

McIntyre, A. (2008). Participatory Action Research. Thousand Oaks, CA: Sage Publications.

McIntyre, A., Chatzopoulos, N., Politi, A., \& Roz, J. (2007). Participatory action research: Collective reflections on gender, culture, and language. Teaching and Teacher Education, 23(5), 748-756. doi:http://dx.doi.org/10.1016/j.tate.2006.12.025

Ministry of Education. (2001). Pasifika Education Research Guidelines. Auckland, NZ: Auckland Uniservice, University of Auckland.

Ministry of Health. (2012). Tupu Ola Moui: Pacific Health Chart Book 2012. Wellington: Ministry of Health. Retrieved from http://www.health.govt.nz/system/files/documents/publications/tupu-ola-moui-pacifichealth-chart-book_1.pdf

Ministry of Health. (2015). Annual Update of Key Results 2014/15: New Zealand Health Survey. Wellington: Ministry of Health. Retrieved from http://www.health.govt.nz/system/files/documents/publications/annual-update-key-results2014-15-nzhs-dec15-1.pdf.

Ministry of Social Development. (2010). The Social Report. Wellington: Ministry of Social Development. Retrieved from http://socialreport.msd.govt.nz/2010/downloads.html 
Montesanti, S. R., Abelson, J., Lavis, J. N., \& Dunn, J. R. (2015). The value of frameworks as knowledge translation mechanisms to guide community participation practice in Ontario CHCs. Social Science \& Medicine, 142, 223-231. doi:http://dx.doi.org/10.1016/j.socscimed.2015.08.024

Moreno-John, G., Gachie, A., Fleming, C. M., Nápoles-Springer, A., Mutran, E., Manson, S. M., \& Pérez-Stable, E. J. (2004). Ethnic Minority Older Adults Participating in Clinical Research: Developing Trust. Journal of Aging \& Health, 93S.

Nomura, M., Makimoto, K., Kato, M., Shiba, T., Matsuura, C., Shigenobu, K., Ishikawa, T, Matsumoto, N, Ikeda, M. (2009). Empowering older people with early dementia and family caregivers: A participatory action research study. International Journal of Nursing Studies, 46(4), 431-441. doi:http://dx.doi.org/10.1016/j.ijnurstu.2007.09.009

Norris, K., Brusuelas, R., Jones, L., Miranda, J., Duru, O., \& Mangione, C. (2007). Partnering with community-based organizations: an academic institution's evolving perspective. Ethnicity \& Disease, 17, S27-32.

Ofanoa, M., Percival, T., Huggard, P., \& Buetow, S. (2015). Talanga: The Tongan Way Enquiry. Sociology Study, 5(4), 334-340.

Ogden, K., Hernandez, B., \& Hidalgo, M. (2010). Payoffs of participatory action research: racial and ethnic minorities with disabilities reflect on their research experiences. Community Development, 2010(41), 21-31. doi:10.1080/15575330903477275

Pack, M., Minster, J., Churchward, M., \& Tanuvasa, A. F. (2013). Evaluation of the implementation and immediate outcomes of the Primary Health Care Strategy: The experiences of Pacific PHOs and Pacific populations Wellington: Health Services Research Centre, Victoria University. Retrieved from http://www.victoria.ac.nz/sog/researchcentres/health-services-researchcentre/publications/reports/Pacific-PHCS-Rpt-FINAL.pdf

Panelli, R., Gallagher, L., \& Kearns, R. (2006). Access to rural health services: research as community action and policy critique. Social Science \& Medicine, 62, 1103-1114.

Paterson, J., Percival, T., Schluter, P., Sundborn, G., Abbott, M., Carter, S., Cowley-Malcolm, E, Borrows, J, Gao, W, the PIF Study Group. (2008). Cohort Profile: The Pacific Islands Families (PIF) Study. International Journal of Epidemiology, 37(2), 273-279. doi:10.1093/ije/dym171

Patten, S., Mitton, C., \& Donaldson, C. (2006). Using participatory action research to build priority setting process in a Canadian regional health authority. Social Science \& Medicine, 63, 1121-1134.

Richardson, K., Jatrana, S., Tobias, M., \& Blakely, T. (2013). Migration and Pacific Mortality: Estimating Migration Effects on Pacific Mortality Rates Using Bayesian Models [Article]. Demography, 50(6), 2053-2073. doi:10.1007/s13524-013-0234-0

Ritchie, J., Bernard, D., Trede, F., Hill, B., \& Squires, B. (2003). Using a participatory action research approach as a process for promoting the health of older people. Health Promotion Journal of Australia, 14(1), 54-60. doi:http://dx.doi.org/10.1071/HE03054

Robinson, O. C. (2014). Sampling in Interview-Based Qualitative Research: A Theoretical and Practical Guide. Qualitative Research in Psychology, 11(1), 25-41. doi:10.1080/14780887.2013.801543

Rozanova, J., Keating, N., \& Eales, J. (2012). Unequal Social Engagement for Older Adults: Constraints on Choice. Canadian Journal on Aging/La Revue canadienne du vieillissement, 31(01), 25-36. doi:doi:10.1017/S0714980811000675

Southwick, M., Kenealy, T., \& Ryan, D. (2012). Primary Care for Pacific People: A Pacific and Health Systems approach: Report to the Health Research Council and the Ministry of Health. Wellington: Pacific Perspectives. Retrieved from 
http://www.health.govt.nz/system/files/documents/publications/primary-care-pacificpeople-pacific-health-systems-approach.pdf

Statistics New Zealand. 2013 Census QuickStats about culture and identity Retrieved from http://www.stats.govt.nz/Census/2013-census/profile-and-summary-reports/quickstatsculture-identity/pacific-peoples.aspx.

Statistics New Zealand and Ministry of Pacific Island Affairs. (2010). Education and Pacific peoples in New Zealand. . Retrieved from http://www.stats.govt.nz/browse_for_stats/people_and_communities/pacific_peoples/pacif ic-progress-education.aspx

Statistics New Zealand and Ministry of Pacific Island Affairs. (2011). Health and Pacific Peoples in New Zealand. Wellington, New Zealand: Authors.

Tamasese, T. K., Parsons, T. L., \& Waldegrave, C. (2014). Pacific perspectives on ageing in New Zealand. Wellington: Family Centre. Retrieved from https://www.massey.ac.nz/massey/fms/Colleges/College of Humanities and Social Sciences/Psychology/HART/publications/reports/Pacific_Elders_NZLSA_2014.pdf?6A68 389EA6EAB37148E4AE22BA963822

Tiatia, J. (1998). Caught between cultures: a new zealand born pacific island perspective. Ellerslie: Christian Research Association.

Tukuitonga, C. (2013). Pacific people in New Zealand. In I. St George (Ed.), Cole's medical practice in New Zealand Wellington, NZ: Medical Council of New Zealand. Retrieved from https://www.mcnz.org.nz/assets/News-and-Publications/Coles/Coles-Medical-Practice-inNew-Zealand-2013.pdf

Vaioleti, T. M. (2006). Talanoa research methodology: A developing position on Pacific research. Waikato Journal of Education, 12, 21-34.

Walker, J., Orpin, P., Baynes, H., Stratford, E., Boyer, K., Mahjouri, N, Paterson, A, Robinson, J, Carty, J. (2013). Insights and principles for supporting social engagement in rural older people. Ageing \& Society, 33(06), 938-963. doi:10.1017/S0144686X12000402

Warburton, J., \& McLaughlin, D. (2005). 'Lots of little kindnesses': valuing the role of older Australians as informal volunteers in the community. Ageing \& Society, 25(05), 715-730. doi:doi:10.1017/S0144686X05003648

Wiles, J., Wild, K, Kepa, M, Peteru, C. (2011). Resilient Ageing in Place: Project Recommendations \& Report: Faculty of Medical and Health Sciences, University of Auckland. Retrieved from http://www.fmhs.auckland.ac.nz/en/soph/about/ourdepartments/social-and-community-health/our-research/resilient-ageing.html.

Worrall, J. (2009). Grandparents and whanau/extended families raising kin children in Aotearoa/New Zealand. A view over time: Grandparents Raising Grandchildren Trust NZ . Retrieved http://www.grg.org.nz/What+we+do/Publications/Research/Research+Report+2009+$+\mathrm{A}+$ view+over+time.html.

\section{Funding}

This work is supported by funding from the Ministry of Business, Innovation and Employment, and the University of Otago National Science Challenge under Grant number UOOX-1508

\section{Notes on the contributors}

Dr Ofa Dewes has a PhD in Public Health and is Affiliate Investigator of the Maurice Wilkins Centre for Molecular Biodiscovery and Research Fellow at the School of Nursing, University of Auckland. Her research interests include Pacific ethnic-specific studies across the life-span especially in health equity, obesity, type 2 diabetes. She is Fiji-born of Rotuman, Tongan, and Tuvaluan ethnicity, with affiliation to Ngati Porou. 
Leon Iusitini is a researcher in the Centre for Pacific Health \& Development in the School of Public Health \& Psychosocial Studies in the Faculty of Health and Environmental Sciences at Auckland University of Technology. He has research interests in public health, psychology, political science, and Pacific peoples in New Zealand. He is currently undertaking doctoral studies.

Professor Stephen Neville is Head of Department (Nursing) and Co-Director of the Centre for Active Ageing at Auckland University of Technology. His research platform is marginalised populations and health workforce development, with a focus on the social aspects of ageing, sexual minority groups, men's health, and supporting the development of an appropriate workforce to support the health and well-being of marginalised groups.

Professor Janis Paterson is Co-Director of the National Institute for Public Health and Mental Health Research in the School of Public Health \& Psychosocial Studies at Auckland University of Technology. Professor Paterson is a developmental psychologist and plays a central role building research collaborations and in the growth and development of a variety of research areas that are aligned with community and government priorities.

Dr El-Shadan Tautolo has a PhD in Public Health, is a Senior Research Fellow and Director for the Centre for Pacific Health \& Development in the School of Public Health \& Psychosocial Studies in Auckland University of Technology. His areas of research expertise are: Pacific health, Public health, Tobacco control, and Longitudinal study design and analysis. He is of Samoan and Cook Islands heritage, and passionate about improving the health and wellbeing of Pacific communities in NZ.

Dr Wendy Wrapson has a PhD in Psychology and is a Senior Research Fellow in the National Institute for Public Health and Mental Health Research in the School of Public Health \& Psychosocial Studies at Auckland University of Technology. Her research is focused on the health and wellbeing of older people and encompasses both quantitative and qualitative research methodologies.

Associate Professor Valerie A. Wright-St Clair is based in the Department of Occupational Science and Therapy, and is Co-director of the Centre for Active Ageing at Auckland University of Technology. Her research projects span studies in social gerontology, including the relationship between older Māori and non-Māori preferred activities and health, older immigrants' community participation, community inclusiveness and loneliness, and quality outcomes in residential aged care. 